Evaluation of respiratory- and postural-induced changes on the ballistocardiogram signal by time warping averaging

This content has been downloaded from IOPscience. Please scroll down to see the full text.

2017 Physiol. Meas. 381426

(http://iopscience.iop.org/0967-3334/38/7/1426)

View the table of contents for this issue, or go to the journal homepage for more

Download details:

IP Address: 131.175.28.198

This content was downloaded on 06/07/2017 at 18:11

Please note that terms and conditions apply.

You may also be interested in:

Robust ballistocardiogram acquisition for home monitoring

O T Inan, M Etemadi, R M Wiard et al.

Non-constrained heart rate variability analysis using a ballistocardiogram

Jae Hyuk Shin, Su Hwan Hwang, Min Hye Chang et al.

A real-time approach for heart rate monitoring using a Hilbert transform in seismocardiograms Mojtaba Jafari Tadi, Eero Lehtonen, Tero Hurnanen et al.

Evaluation of respiratory influences on left ventricular function parameters extracted from echocardiographic acoustic quantification

Enrico G Caiani, Maurizio Turiel, Sabrina Muzzupappa et al.

Extracting respiratory information from seismocardiogram signals acquired on the chest using a miniature accelerometer

Keya Pandia, Omer T Inan, Gregory T A Kovacs et al.

Ballistocardiography in sitting and horizontal positions

J Alametsä, J Viik, J Alakare et al.

Non-constrained monitoring of systolic blood pressure on a weighing scale

Jae Hyuk Shin, Kang Moo Lee and Kwang Suk Park

Quantification of respiratory influence on LV

Enrico G Caiani, Maurizio Turiel, Sabrina Muzzupappa et al.

Non-invasive cardiac output trending during exercise recovery on a bathroom-scale-based BCG

O T Inan, M Etemadi, A Paloma et al. 


\title{
Evaluation of respiratory- and postural- induced changes on the ballistocardiogram signal by time warping averaging
}

\author{
A Martín-Yebraa ${ }^{1,2}$, F Landreani ${ }^{1}$, C Casellato ${ }^{1}$, E Pavan $^{1}$, \\ P-F Migeotte ${ }^{3}$, C Frigo ${ }^{1}$, J P Martínez ${ }^{2,4}$ and E G Caiani ${ }^{1,5}$ \\ ${ }^{1}$ Dipartimento di Elettronica, Informazione e Bioingegneria, Politecnico di Milano, \\ Milano, Italy \\ 2 BSICoS Group, Instituto de Investigación en Ingeniería de Aragón (I3A), IIS \\ Aragón, Universidad de Zaragoza, Zaragoza, Spain \\ 3 Laboratory of Physics and Physiology, Université Libre de Bruxelles, Bruxelles, \\ Belgium \\ ${ }^{4}$ Centro de Investigación Biomédica en Red-Bioingeniería, \\ Biomateriales y Nanomedicina, Zaragoza, Spain \\ E-mail: enrico.caiani@polimi.it and albapilar.martin@polimi.it
}

Received 4 January 2017, revised 5 May 2017

Accepted for publication 12 May 2017

Published 27 June 2017

\begin{abstract}
Objective: The aim of this work was to evaluate the potential changes in the ballistocardiogram (BCG) signal induced by different respiratory patterns and posture, by using the dynamic time warping (DTW) technique. Approach: BCG signals were recorded in a group of 20 healthy volunteers, simultaneously with an electrocardiogram (ECG). Two recordings, one in a supine (SUP) and one in a standing (ST) position, including spontaneous breathing, two $1 \mathrm{~min}$ apneas (at full and empty-lungs, respectively) and $30 \mathrm{~s}$ of Valsalva, were analyzed. A warped averaged waveform was computed for each phase, from which amplitude and temporal parameters were extracted to characterize each condition. Main results: Variations were found in both amplitude and duration of BCG-derived parameters among manoeuvres, especially when compared to spontaneous breathing, suggesting a complex interaction between intrathoracic pressure changes acting on venous return, together with the autonomic nervous system modulation on heart rate. The effect of a hydrostatic pressure gradient elicited by postural conditions was also evident. Significance: Posture and respiratory manoeuvres affect the BCG signal in different ways, probably as a result of changes induced in preload and afterload. This supports the need to
\end{abstract}

\footnotetext{
${ }^{5}$ Author to whom any correspondence should be addressed.

Politecnico di Milano, Dipartimento di Elettronica, Informazione e Bioingegneria Piazza Leonardo da Vinci 32, 20.133, Milano.
} 
define separate normality ranges for each posture and/or breathing conditions, as well as the importance of applying specific manoeuvres to highlight any pathological response in the computed BCG parameters.

Keywords: ballistocardiogram (BCG), signal averaging, dynamic time warping (DTW), cardiac monitoring, respiration

(Some figures may appear in colour only in the online journal)

\section{Introduction}

Ballistocardiography is a non-invasive technique that measures the heartbeat-induced mass movements of circulating blood and of the heart itself, generated by the acceleration of blood as it is ejected and moved to the aorta and the large vessels (Gubner et al 1953). It is represented by a succession of systolic (H, I, J, K) and diastolic (L, M, N) waves, describing the forces associated with the shifting of the centre of mass of the body throughout the cardiac cycle (Pinheiro et al 2010). The ballistocardiogram (BCG) signal has recently been reconsidered as a useful technique in the characterization of cardiac performance (Inan et al 2015), offering a simple, efficient and affordable solution for home e-health monitoring (Javaid et al 2016), potentially representing an important practice in the prevention and early detection of cardiac-related diseases.

Temporal parameters, such as the RJ interval (the time interval between the occurrence of the R-peak on the ECG signal and the corresponding J wave on the BCG), have been proposed as a surrogate for the pre-ejection period (PEP), to assess cardiac contractility non-invasively (Inan et al 2008, Etemadi et al 2011, Javaid et al 2016). On the other hand, the amplitude of the BCG signal, especially in systolic waves, is mainly correlated with cardiac output, cardiac force, and velocity of ejection (Starr et al 1939), and has been found to be an indicator of myocardial contraction force (Starr 1955, 1965).

However, the BCG quasi-periodic signal is characterized by a large inter-beat variability in its morphology (both in duration and in amplitude), mainly related to the respiratory activity and mediated by the autonomic nervous system, thus affecting both preload and afterload by changes in intrathoracic pressure.

In order to extract valuable information from the BCG signal, two approaches have been proposed: (1) to extract beat-by-beat series from short-duration $(\geqslant 3 \mathrm{~min})$ recordings in order to characterize time and frequency parameters of heart-rate variability (Shin et al 2011, Zhu et al 2012); (2) to average consecutive beats acquired over shorter sequences ( $<1 \mathrm{~min})$ in order to obtain a characteristic waveform from which to derive indices of cardiac performance (Etemadi et al 2011, He et al 2011).

With regard to the averaging of biological signals, three approaches are available: synchronous, template-based, and non-linear techniques. Synchronous averaging is extensively used when a stimulus is present and the system's response is supposed to be stationary, where the waveforms are averaged after being aligned with respect to a reference point (Cohen 1986). The template-based approach is based on a priori selection of a reference waveform (i.e. the template) which is used to filter out non-similar waveforms from the signal, resulting in the averaging of the remaining similar ones (Aunon et al 1980). Finally, non-linear averaging techniques have been proposed to overcome limitations of the previous methods, in particular when dealing with quasi-periodic biological signals, whose morphology varies both in amplitude and in duration.

In particular, the dynamic time warping (DTW) technique, originally proposed with success for speech recognition (Sakoe and Chiba 1978), has been already applied to an echo-derived 
left ventricular volume signal for the assessment of its function (Caiani et al 2002). The potential of this technique is that it allows the averaging operation to be performed after finding the best alignment between consecutive waveforms, thus compensating for temporal distortions without any a priori information.

We hypothesised that the use of the DTW algorithm could be successfully applied to the BCG signal to obtain an average waveform from a sequence of consecutive beats, from which to extract amplitude and temporal parameters to characterize cardiac performance.

In order to define a proper acquisition protocol that could then be implemented in a homemonitoring scenario, our aim was to evaluate the potential changes in the BCG signal induced by different postural conditions, as well as during different respiratory manoeuvres.

\section{Materials and methods}

\subsection{Study protocol and population}

Three-orthogonal components of the BCG signal, denoted as AP (Anterior-Posterior), LR (Left-Right, mediolateral), and HF (Head-Foot, longitudinal), were acquired (sampling frequency $960 \mathrm{~Hz}$ ) by using a multicomponent biomechanical force plate, based on 3D piezoelectric load cells (Type $9286 \mathrm{~B}$, Kistler ${ }^{\circledR}, 600 \times 400 \mathrm{~mm}$ ), placed on the floor with its top surface $40 \mathrm{~mm}$ above floor level. The experimental set-up also included a rigid wooden platform (length 2100 , width 400 , height $40 \mathrm{~mm}$ ) placed over the force plate, with its centre corresponding to the centre of the force plate and parallel to the horizontal plane, in order to avoid any contact with the floor. Forces induced by the body mass micro-displacements were directly transferred to the force plate, this being able to capture magnitude changes as small as $0.01 \mathrm{~N}$. An offset-reset of the force platform was performed at the onset of each acquisition, allowing both the body weight and the wooden platform weight (i.e. the continuous signal component) to be automatically removed.

Simultaneously, a standard three-lead ECG signal was collected by a portable system (Porti TMSiTM, sampling frequency $2048 \mathrm{~Hz}$ ). The ECG and BCG signals were synchronized by using a TTL function generator that sent a signal simultaneously acquired by both systems.

Subjects were first asked to stay in a supine (SUP) position, with their centre of mass aligned with the central point of the force plate (figure 1(a)), and then in a standing (ST) position, with their feet centrally positioned (figure 1(b)). Our acquisition protocol included two consecutive recordings (SUP and ST, respectively), each consisting of:

(a) 5 min at resting conditions;

(b) 3 preparatory deep breaths;

(c) 1 min apnea at end-inspiration (Full-lung apnea);

(d) 1 min of normal breathing;

(e) 3 preparatory deep breaths;

(f) 1 min apnea at end-expiration (Empty-lung apnea);

(g) 1 min of normal breathing;

(h) $30 \mathrm{~s}$ of Valsalva manoeuvre.

Subjects were asked to stay quiet and not to make any voluntary movements throughout the acquisition period, in order to minimize motion artefacts and to ensure that the signal oscillations were mainly due to mass motion inside the body.

The analysed population was a group of 20 healthy volunteers (12 females) whose main characteristics are summarised in table 1. 


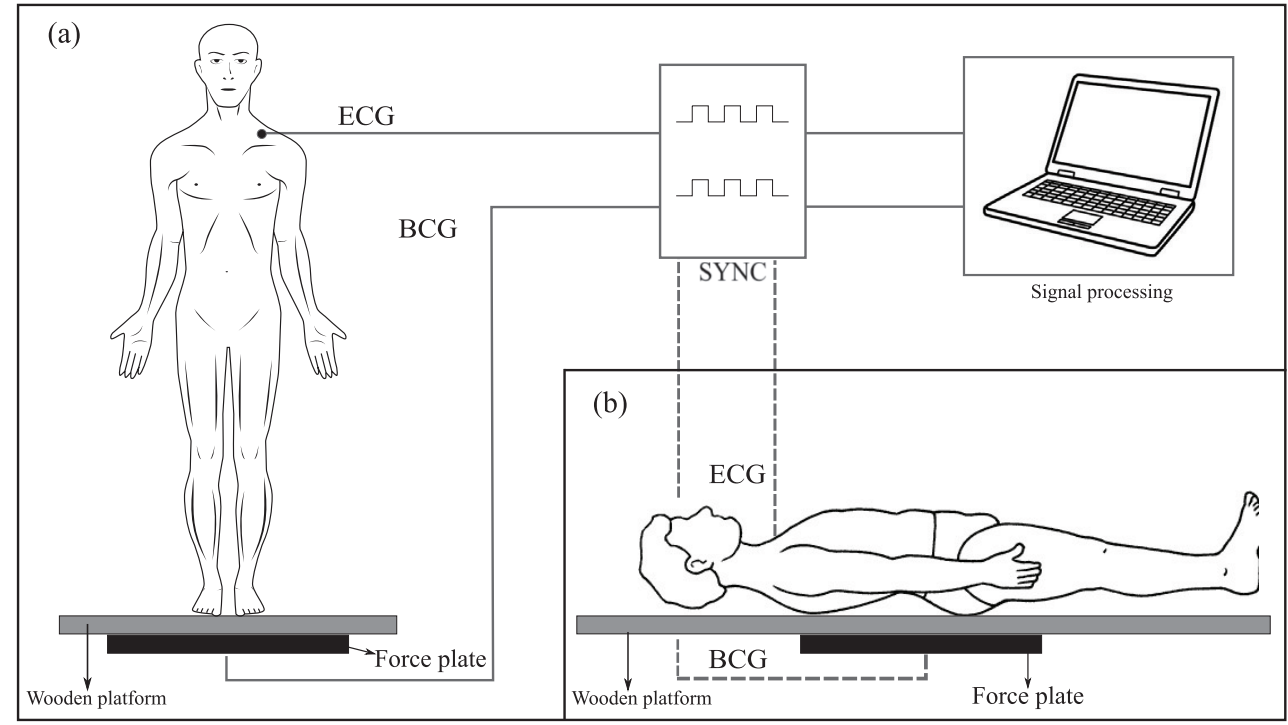

Figure 1. Schematic of the experimental set-up for the subject in standing position (a) and supine (b).

Table 1. Study population characteristics expressed as mean \pm std.

\begin{tabular}{lll}
\hline Variable & Males & Females \\
\hline Age (years) & $32.9 \pm 9.0$ & $28.2 \pm 4.8$ \\
Weight $(\mathrm{kg})$ & $72.9 \pm 10.5$ & $53.9 \pm 8.3$ \\
Height $(\mathrm{m})$ & $1.79 \pm 0.08$ & $1.62 \pm 0.06$ \\
BMI $\left(\mathrm{kg} \mathrm{m}^{-2}\right)$ & $22.8 \pm 4.06$ & $20.6 \pm 2.13$ \\
\hline
\end{tabular}

BMI: body mass index.

All participants gave written informed consent to participate in the study. The experimental procedures involving healthy volunteers described in this paper were in agreement with the principles outlined in the Helsinki Declaration of 1975, as revised in 2013. Ethical committee approval of the Université Libre de Bruxelles, Hôpital Erasme, was obtained prior to the study.

\subsection{Pre-processing}

The pre-processing stage included automatic QRS detection from the ECG signal using a wavelet-based ECG delineator (Martinez et al 2004), followed by ECG-BCG synchronization using the trigger signal. The BCG signal was then filtered (band pass, 0.1-30 Hz, Butterworth filter, order 8) in order to remove baseline wander, noise, and other out-of-band components (high-frequency motion artifacts).

Figure 2 shows an example of the three-orthogonal filtered components of the reaction force from a representative subject, in supine (upper panel) and standing (bottom panel) positions, synchronized with the ECG signal. In both postures the best signal-to-noise ratio, and the most informative content showing the quasi-periodic heart-induced activity, was visible in the longitudinal head-to-foot (HF) component. Based on this consideration, together with the fact that it has been the most widely-studied component and the only one for which an interpretation has been provided, we focused our attention on this component only. 

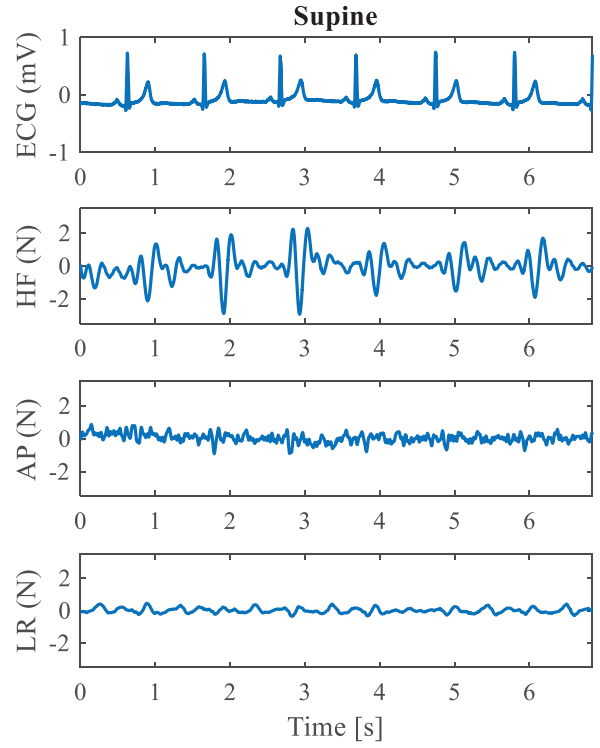
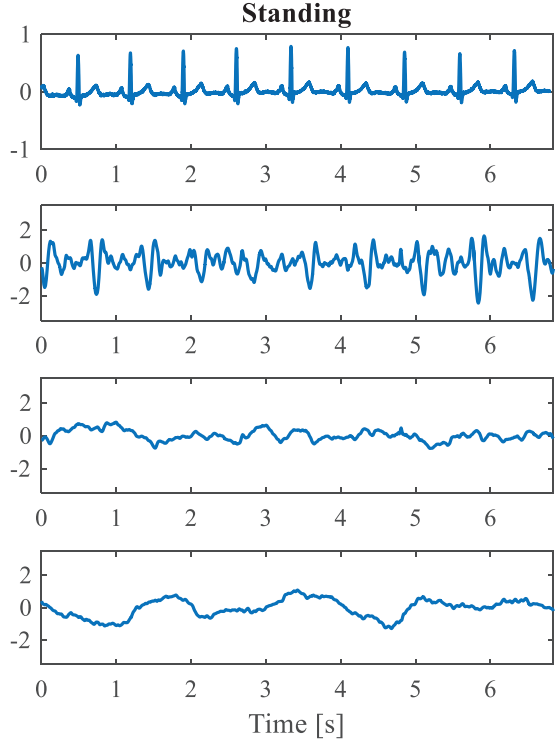

Figure 2. Example of synchronized ECG and the three components of the BCG signal: HF (head-to-foot), AP (antero-posterior) and LR (left-to-right) components, in both supine (left) and standing (right) positions.

Finally, BCG segments of the acquisition relevant to phases (a), (c), (f) and (h) of the protocol were extracted. R-peak events on the ECG were utilized to delimit each BCG cardiac cycle.

\subsection{Dynamic time warping}

The DTW averaging technique (Sakoe and Chiba 1978) comprises the iterating of two consecutive steps performed on pairs of BCG waveforms, each representing one cardiac cycle, according to a binary tree combination procedure:

1. the calculation of the time-warping function (TWF) to perform the best alignment between their temporal axes, and;

2. the computation of a new waveform by mixing and averaging the values from the two original cycles, according to the TWF.

2.3.1. Calculation of the TWF. A more detailed description of the computation of the TWF can be found elsewhere (Caiani et al 2002). Summarizing, given two BCG waveforms, $S_{x}(n)$ and $S_{y}(m)$, with different durations ( $1 \leqslant n \leqslant N$ and $1 \leqslant m \leqslant M$, respectively) but equally sampled, the temporal correspondence between samples of $S_{x}$ and $S_{y}$ is defined by the monotonic and continuous TWF, matching the time axis $n$ of the waveform $S_{x}$ in the time axis $m$ of the waveform $S_{y}$, and vice-versa. Figure 3 shows an example of the TWF represented in the plane $(n, m)$, with the patterns $S_{x}$ and $S_{y}$ on the $n$ and $m$ axes, respectively.

To calculate the TWF, the following measure of dissimilarity (i.e. the local cost) between the normalised waveforms $S_{x}$ and $S_{y}$ was utilised, as described in Caiani et al (2002), taking into account their amplitude and first derivative differences:

$$
d(n, m)=k_{1}\left|S_{x}(n)-S_{y}(m)\right|+k_{2}\left|\dot{S}_{x}(n)-\dot{S}_{y}(m)\right|
$$

with $k_{1}=k_{2}=1$, and $\dot{S}$ representing the first normalized derivative. The value of $d(n, m)$ is computed at every point of the warping plane $(n, m)$ that lies inside the zone of the plane $(n, m)$ 


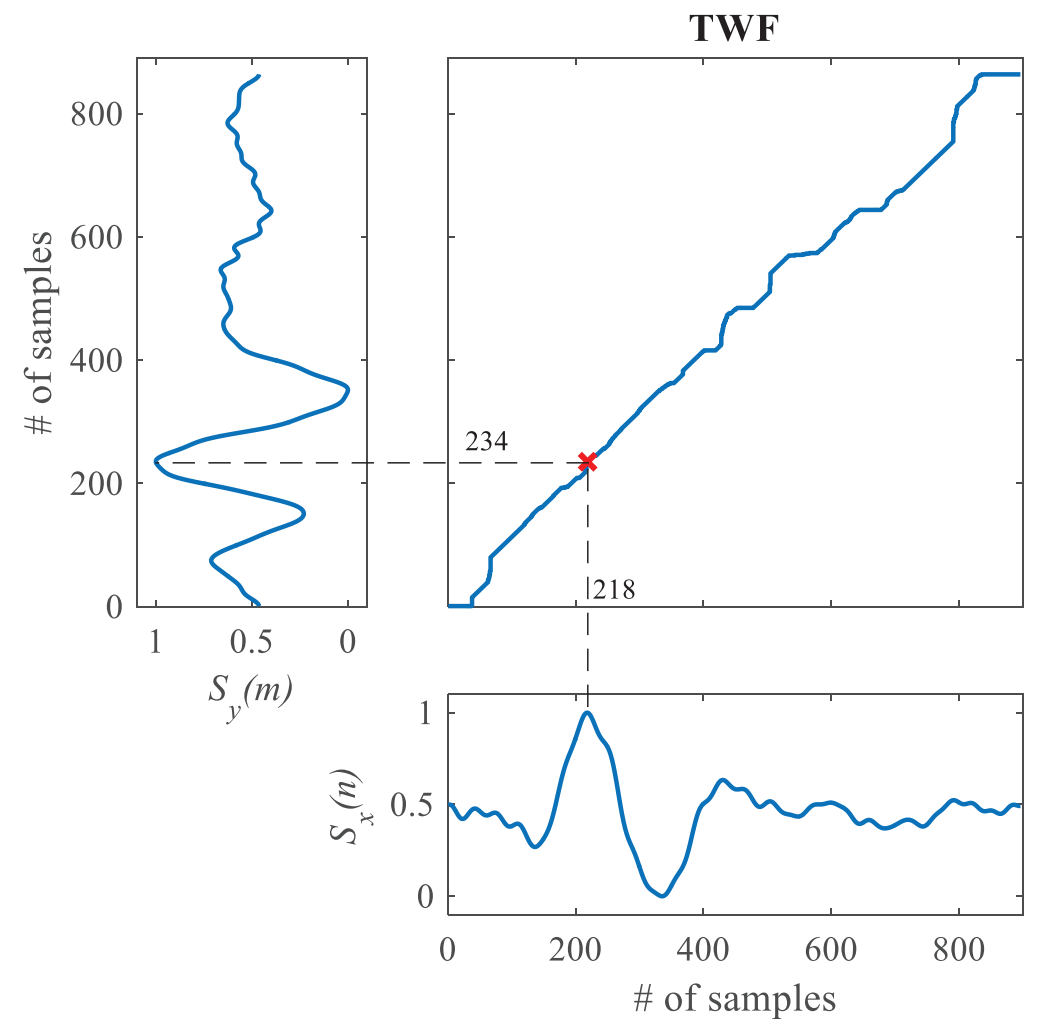

Figure 3. Example of the computed time-warping function (TWF) that best aligns the two consecutive BCG normalized waveforms, here denoted as $S_{x}(n)$ and $S_{y}(m)$. The red cross represents the correspondence between the two J positive waves in both waveforms (samples $n=218$ and $m=234$, respectively).

around the main diagonal, defined as $|n-m| \leqslant r$, where $r=10+|N-M|$, thus preventing excessive adjustment of the time axes.

Then, given the local dissimilarity $d(n, m)$, by a dynamic programming algorithm (Sakoe and Chiba 1978) a cost matrix $D$ was computed using the symmetric form without slope constraints (Sakoe and Chiba 1978), where $D(n, m)$ represents the minimum cumulative cost to reach the point $(n, m)$ starting from $(1,1)$ :

$D(n, m)=\min \{D(n, m-1)+d(n, m), D(n-1, m)+d(n, m), D(n-1, m-1)+2 d(n, m)\}$

with the initial condition of $D(1,1)=2 d(1,1)$ and boundary conditions of $D(n, 1)=D(n-1,1)+d(n, 1)$ and $D(1, m)=D(1, m-1)+d(1, m)$. The TWF is finally derived as the path with minimum cost from $(1,1)$ to $(N, M)$.

2.3.2. Computation of the averaged waveform. The warped average (WA) between two consecutive BCG cycles was computed according to the TWF as described in Caiani et al (2002), resulting in a waveform of duration equal to $(N+M) / 2$. The final WA is computed from a set of $L$ consecutive cycles, with $L$ being an integer to the power of two. Waveforms are grouped in $L / 2$ pairs, resulting in $L / 2$ WAs being computed. Then, this step is iterated until just one final WA beat is obtained. 


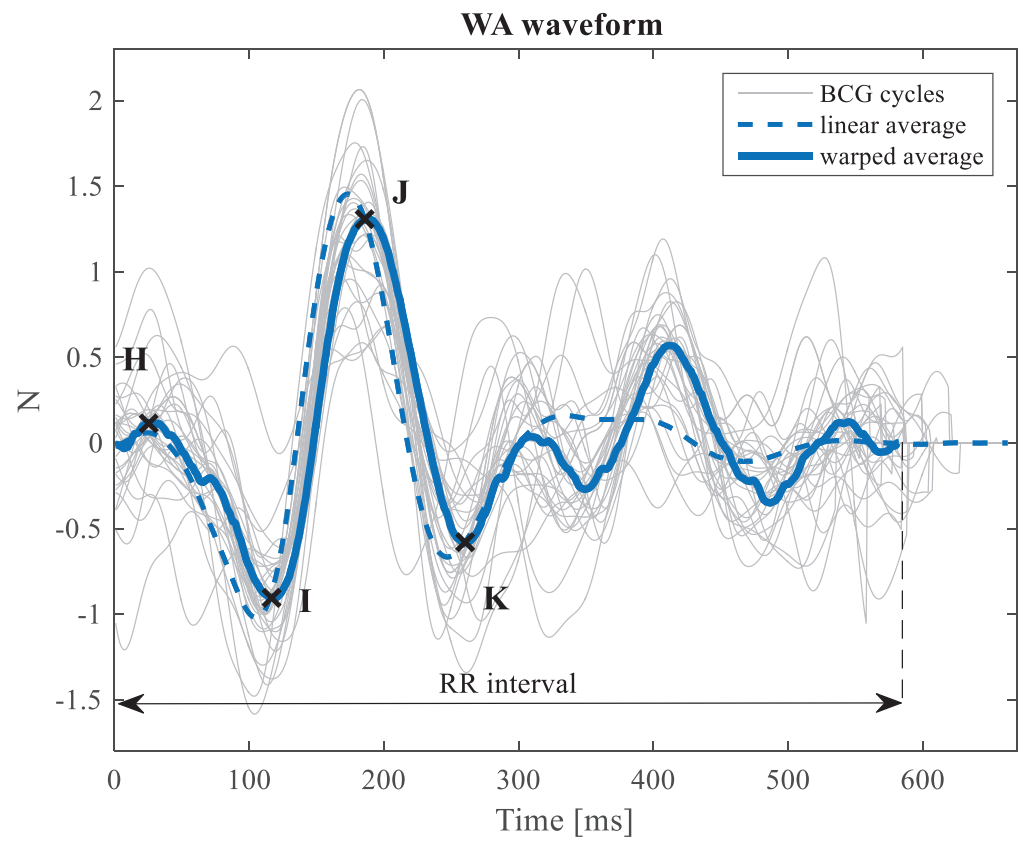

Figure 4. Example of a sequence of $32 \mathrm{BCG}$ waveforms (light grey) obtained in one subject, with the results of their synchronous averaging (blue dashed line) and of the DTW averaging (blue line).

To compare the effects of the different manoeuvres, $L=32$ consecutive beats were considered for each phase of the protocol (number limited by the shortest phase that is the $30 \mathrm{~s}$ Valsalva manoeuvre stage). Figure 4 shows an example of the original 32 consecutive beats obtained in a subject in a standing position during the resting period, together with the corresponding synchronous averaging (blue dashed line) and the final warped average (blue line). It can be seen how the characteristic peaks of the BCG signal were maintained, especially during the diastolic phase, by the proposed DTW averaging technique, despite differences in both amplitude and duration among the considered waveforms. As expected, amplitude and temporal position of the H, I, J, K peaks appeared dependent on the utilized averaging approach.

\subsection{Fiducial points detection}

For each phase of the protocol, that is, resting, full-lung apnea (Full), empty-lung apnea (Emp) and Valsalva (Val), the respective WA waveform was computed.

As the WA represents the temporal average of the 32 consecutive beats, the duration of the template defines the average RR interval. Systolic fiducial points were then identified on each final WA waveform, as shown in figure 4: according to Pinheiro et al (2010) the $\mathrm{H}$ wave represents the first positive peak, synchronous with the isovolumetric contraction; the I wave, which is the first negative wave at the onset of ejection (early systole), corresponds to blood acceleration in the ascending aorta and pulmonary arteries; the J wave, the main positive wave occurring late in systole, corresponds to blood acceleration when ejected into the descending aorta; and finally, the $\mathrm{K}$ wave corresponds to the second negative deflection associated with the descending slope of the femoral pulse, synchronized with the second heart's sound, and so with closure of the semilunar valves. The RK interval represents the systolic phase, while the complementary KR interval represents the diastolic period. 

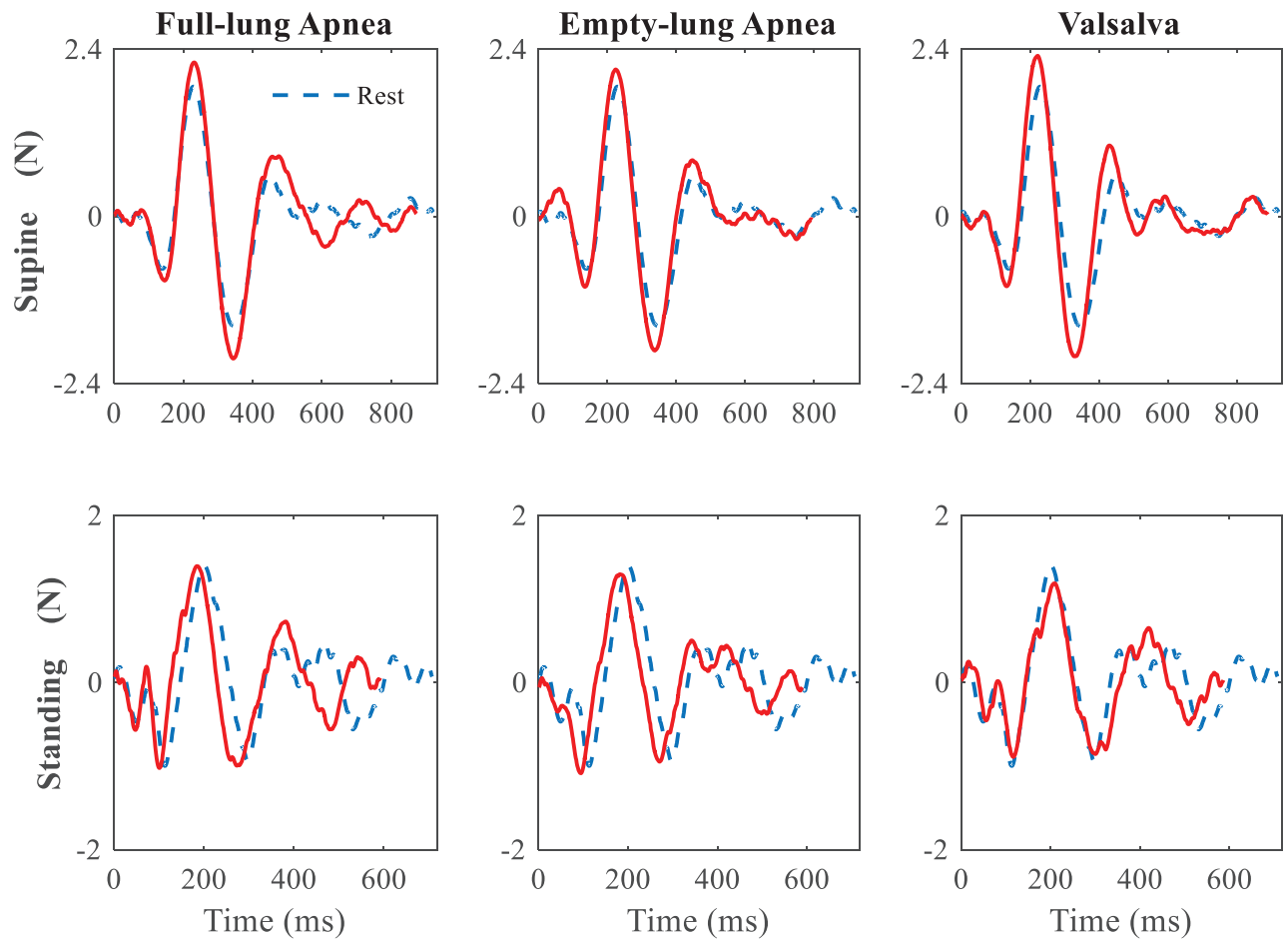

Figure 5. An example of warped average waveforms computed from one subject at each respiratory condition in both supine (upper panels) and standing (bottom panels) positions. The blue dashed line corresponds to the WA at Rest condition.

The following temporal parameters were extracted and normalized by the corresponding RR duration, in order to describe the relative time occurrence of the main BCG events: RI, RJ, RK and IK sub-intervals. In addition, the peak-to-peak amplitude parameters $A_{\mathrm{HI}}, A_{\mathrm{IJ}}$ and $A_{\mathrm{JK}}$ were computed. They are inertial forces in nature, so it was deemed reasonable to normalize them by the subject's body mass index (BMI).

\subsection{Statistical analysis}

Due to the non-Gaussian distribution of the temporal and amplitude parameters, data are presented as median and 25th and 75th percentiles, unless otherwise specified. To evaluate changes in BCG signals induced by the imposed breathing manoeuvres, a non-parametric paired Wilcoxon rank test was applied to both the temporal (RR, RI, RJ, RK and IK) and the amplitude parameters $\left(A_{\mathrm{HI}}, A_{\mathrm{IJ}}\right.$ and $\left.A_{\mathrm{JK}}\right)$ to compare the resting condition and each of the breathing manoeuvres: full-lung apnea, empty-lung apnea, and Valsalva. To test the effects of posture, a non-parametric paired Wilcoxon rank test was also applied between the corresponding SUP and ST values for each parameter. Additionally, the same test was applied to test the null hypothesis that empty- and full-lungs apneas generated a similar response on the examined parameters. The null hypothesis was rejected when $p \leqslant 0.05$. 

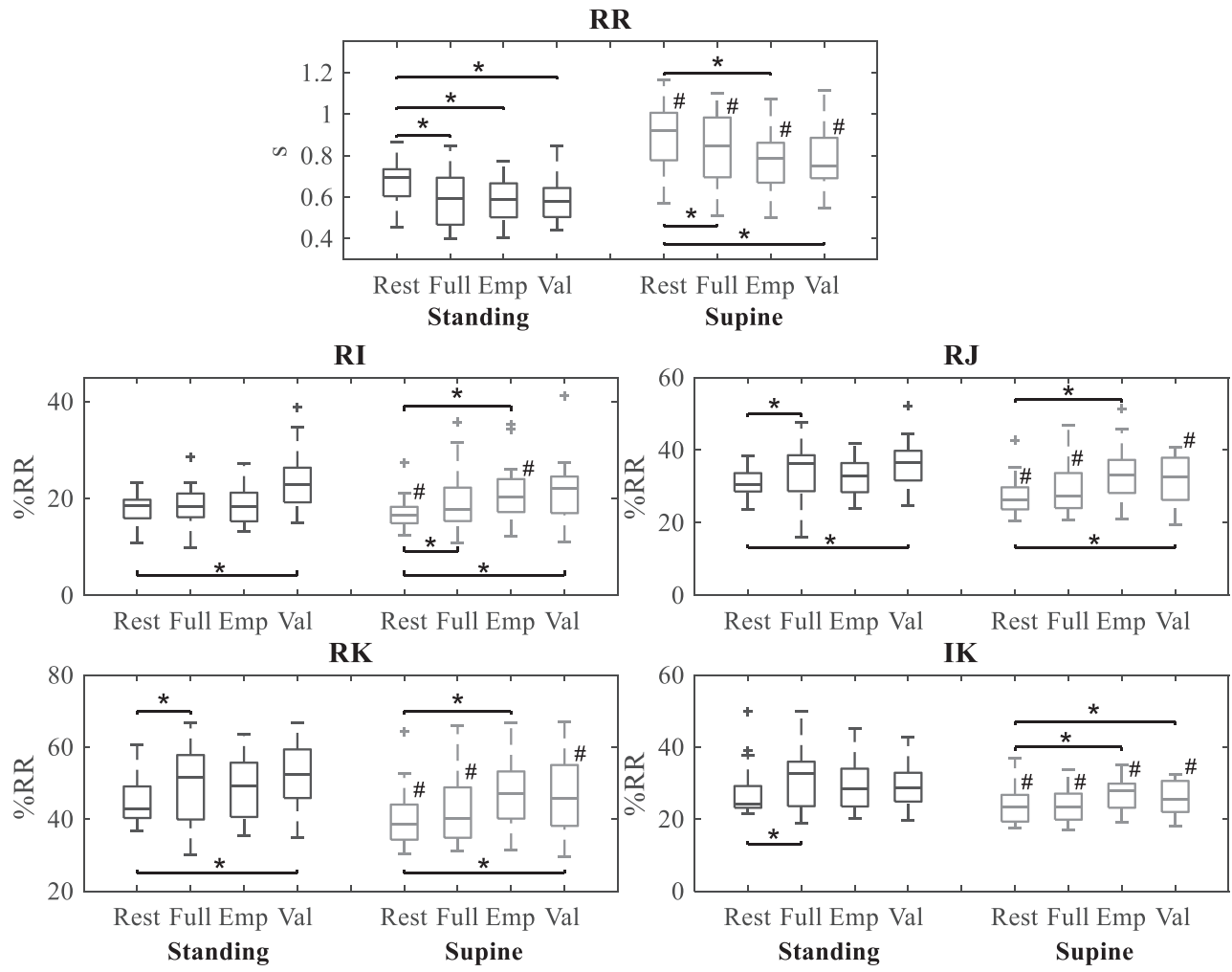

Figure 6. Results of RR and normalized sub-intervals obtained in the normal population of 20 subjects represented as Whisker plots. * $p \leqslant 0.05$ versus Rest; \#: $p \leqslant 0.05$ SUP versus ST.

\section{Results}

An example of the WA computed in one subject and during each of the studied manoeuvres and postures is shown in figure 5. In each panel, the red waveform corresponding to each different tested condition is superimposed on the waveform corresponding to the spontaneous breathing (Rest) condition, represented as a blue dashed line. In this case, it is possible to observe how the RR duration was reduced in the ST posture compared to the SUP posture. In all cases it was also longer in Rest than in the other breathing manoeuvres, as expected from physiology.

The results of the studied normal population in terms of normalized BCG sub-intervals and peak-to-peak amplitudes are shown in figures 6 and 7, respectively, while their absolute values are reported in table 2 .

As previously observed, the RR interval was shortened for each respiratory manoeuvre with respect to the Rest condition, with significantly shorter values in the ST posture than in SUP, as expected (see figure 6, upper panel).

Regarding BCG-derived normalized sub-intervals, in the SUP posture the respiratory manoeuvres affected the $\mathrm{I}, \mathrm{J}$ and $\mathrm{K}$ time occurrence by prolonging the corresponding temporal parameters in empty-lung apnea $(\mathrm{RI}+19 \%, \mathrm{RK}+15 \%, \mathrm{RJ}+16 \%, \mathrm{IK}+11 \%)$ and Valsalva (RI $+26 \%, \mathrm{RK}+13 \%, \mathrm{RJ}+21 \%, \mathrm{IK}+11 \%)$, expressed as a percentage variation compared to the Rest value, while full-lung apnea affected only RI (+7\%). 


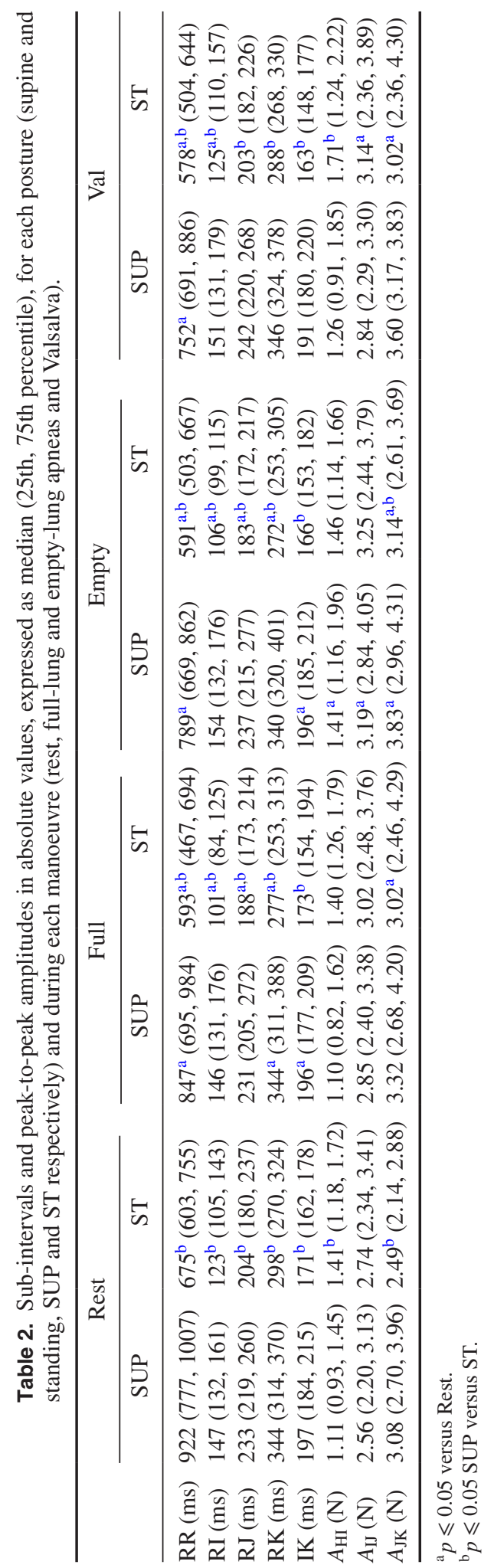



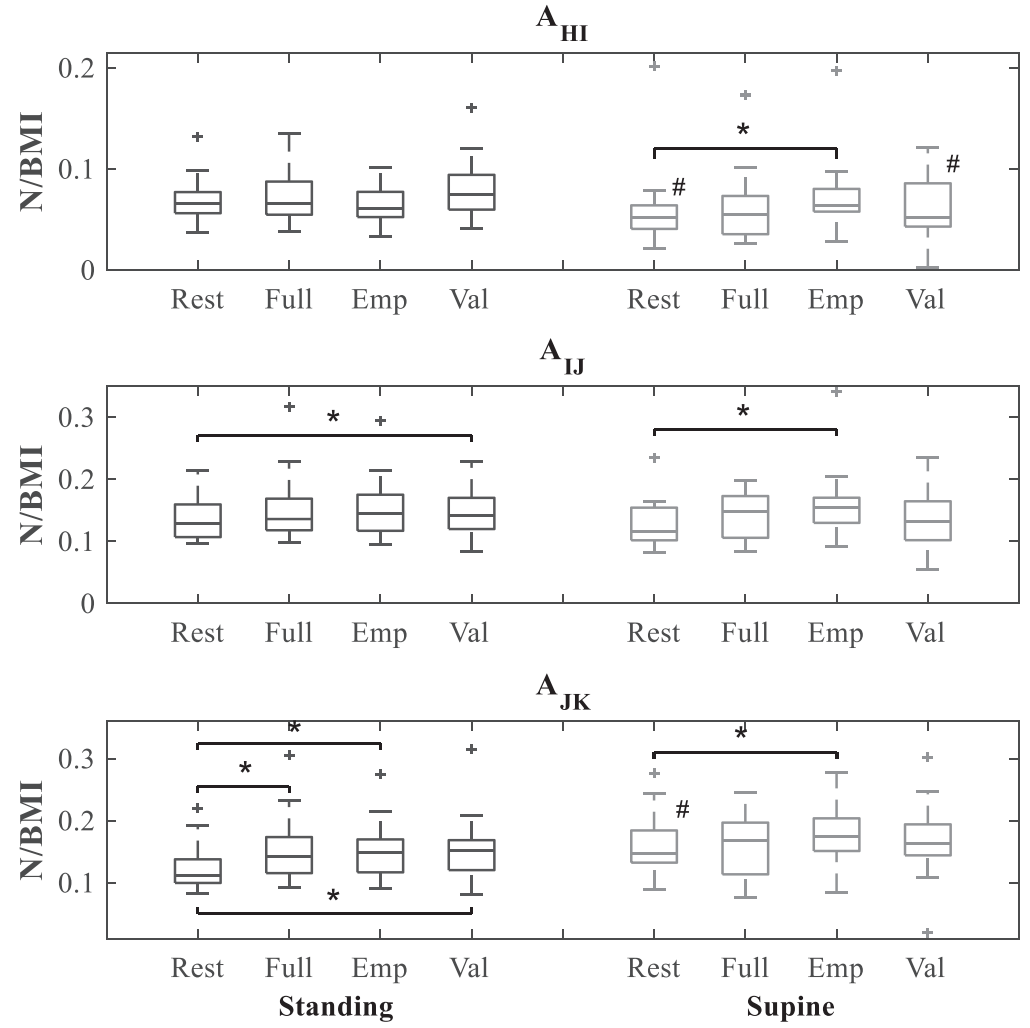

Figure 7. Results of normalized peak-to-peak amplitudes obtained in the normal population of 20 subjects represented as Whisker plots. *: $p \leqslant 0.05$ versus Rest; $\#: p \leqslant 0.05$ versus Standing.

In the ST posture, empty-lung apnea did not significantly modify any parameter, while full-lung apnea prolonged all sub-intervals (RK $+9 \%$, RJ $+9 \%$, IK $+10 \%)$ except RI. In the same posture the Valsalva manoeuvre prolonged all sub-intervals $(\mathrm{RI}+24 \%, \mathrm{RK}+17 \%$, RJ $+11 \%$ ) except IK. When comparing SUP versus ST conditions, the majority of parameters resulting were shorter for SUP. This was particularly evident for the IK interval, which was always shorter in SUP than in ST.

Finally, when comparing the two apneas (Full and Emp), all temporal parameters (RR, RI, RJ, RK, IK) were significantly different between these two conditions $(p<0.05$ in all cases) in the supine position, whereas no significant differences were found while standing.

As regards normalized peak-to-peak amplitudes, in SUP the only respiratory manoeuvre that affected all the indices compared to Rest was the empty-lung apnea $\left(A_{\mathrm{HI}}+25 \%\right.$, $\left.A_{\mathrm{IJ}}+17 \%, A_{\mathrm{JK}}+17 \%\right)$.

In the ST posture, $A_{\mathrm{HI}}$ was unchanged, $A_{\mathrm{IJ}}$ increased by $13 \%$ only with Valsalva, while $A_{\mathrm{JK}}$ increased by $20 \%$ as a result of each manoeuvre. Compared to ST, the main differences in SUP were found in Rest, with a decrease in $A_{\mathrm{HI}}$ and an increase in $A_{\mathrm{JK}}$ peak-to-peak amplitudes. In addition, $A_{\mathrm{HI}}$ was found to decrease during the Valsalva manoeuvre. 


\section{Discussion and conclusion}

Ballistocardiography was originally proposed as a diagnostic tool to study cardiac mechanics and obtain information about cardiac contractility by measuring the shifting in the body mass centre due to displacements of the heart, as well as of the blood masses in the vessels due to cardiac contractions (Starr et al 1939, Gubner et al 1953, Starr 1955, 1965). However, the limited technology available during its early development and the large inter-subject variability of the signal impeded the achievement of a generalized usage.

More recently, new developments in accelerometer technology and hardware miniaturization have led to the reconsideration of BCG as a potentially useful non-invasive methodology with which to characterize cardiac performance (Inan et al 2015). In fact, this technique potentially offers a simple, efficient and affordable solution for home e-health monitoring as it can be embedded in commonly used objects (e.g. weight scales, bed, chair). In particular, the RJ interval has been proposed as a surrogate for the pre-ejection period (PEP) (Inan et al 2009, Etemadi et al 2011, Javaid et al 2016), a non-invasive indirect measurement of cardiac contractility (Lewis et al 1977), and, combined with photoplethysmography (PPG), both I and $\mathrm{J}$ time occurrences have been shown to be an accurate estimate of arterial stiffening (Wiard 2012).

At this point, it becomes important to define a range of values from a normal population for both temporal and amplitude parameters that can be extracted from the BCG, and to study their changes related to posture or respiratory manoeuvres. This information could reveal its utility in the definition of a specific acquisition protocol, and reveal pathologic or physiologically-induced alterations.

In our work, we aimed at studying the BCG signal obtained by a force platform in a population of normal subjects in different cardiac preload and afterload conditions, as well as different autonomic control activities, by means of changes in posture (supine and standing) and by different simple respiratory protocols (normal breathing, apnea and Valsalva).

Indeed, respiration is one of the strongest modulators of cardiac function, as a consequence of changes in intra-thoracic pressure and systemic venous return (Guyton and Hall 2006). In particular, with the Valsalva manoeuvre a sequence of rapid changes in preload and afterload stress is induced. More precisely, it has a direct influence on the pattern of the cardiovascular system, whose response has been reported to be subdivided into four phases (Hamilton et al 1936, Looga 2005). In the early phases of strain (I-II), the increase in intra-thoracic pressure results in a concomitant increase in both heart rate and blood pressure (Porth et al 1984); this is followed by a an increase in parasympathetic activity after strain release (phases III and IV), resulting in a decrease in heart rate.

Apneas performed at end-inspiration and at end-expiration are related to different intrathoracic pressures and non-modulated venous return, providing the possibility to acquire the BCG signal in a relatively steady-state condition. However, the cardiovascular response during these manoeuvres has been reported to be variable over time. Javorka and colleagues showed how an empty-lung apnea induced an initial tachycardia in the first $10 \mathrm{~s}$ approximately, followed by a compensatory decrease in heart rate, evaluated in 24 young healthy volunteers while in a sitting position (Javorka et al 2001). The results of our work revealed that the empty-lungs apnea resulted in a different outcome in temporal parameters than for full-lung apnea, but only in the supine position. This could be due to the fact that in standing, the initial control condition before the apnea was already manifesting a sympathetic activation (median RRrest $=675 \mathrm{~ms}$ in standing, versus $922 \mathrm{~ms}$ in supine), thus limiting the additional effects of the provoking manoeuvre. 
Our results confirmed that acquisition of BCG from a piezoelectric load cells force platform was feasible in all subjects, and signal quality allowed for further analysis and automated parameters extraction in both examined postural conditions.

The acquired BCG is a quasi-periodic signal characterized by a large inter-beat variability in morphology (both in duration and in amplitude). Based on these signal characteristics, DTW was selected as the most appropriate averaging method and was applied to 32-beat sequences obtained during the different breathing manoeuvres imposed. This number of consecutive beats was determined as a compromise between the requirement of acquiring an adequate number of waveforms to be included in the warped averaging in order to improve the signal-to-noise ratio, and the need to keep as short as possible the acquisition in an hypothetical user-driven scenario in which the subject has to maintain the suggested posture for a limited time, both during spontaneous breathing and respiratory manoeuvres. In particular, during the Valsalva manoeuvre, this choice resulted in the focusing of our analysis on the effects relevant to the early phases of the cardiovascular response. Also, for apneas, the choice to limit our analysis to the first 32 beats acquired during this manoeuvre led to a predominance of the initial tachycardia in the observed results. To our knowledge, this is the first time that a DTW technique has been applied to the BCG signal with the goal of generating normality ranges in different postural and breathing manoeuvres.

The DTW method resulted in an averaged waveform in both duration and amplitude from which both temporal and peak-to-peak amplitude parameters were automatically extracted. In order to obtain a range of comparable results representative of the studied normal population, two different normalizations were introduced. For temporal indices, each sub-interval parameter was normalized by the corresponding heart beat duration; for amplitude indices, each peak-to-peak parameter was normalized by the subject BMI. Normalization by the heartbeat duration allowed for the pooling of subjects for comparison of temporal BCG parameters between SUP and ST postures, despite heart rate being found to increase while in a standing posture, as well as between the resting spontaneous respiration and each breathing manoeuvre. Similarly, normalization by the BMI was intrinsically corrected for possible inter-subject variability due to different stroke volume and cardiac output, thus allowing cumulative analysis without other distinctions (i.e. sex, body composition) (Collis et al 2001).

Obtained results showed modifications in both amplitude and duration of BCG-derived parameters among the respiratory manoeuvres, especially when compared to spontaneous breathing, but being also dependent on the assumed posture. The prolongation of the RJ interval during Valsalva, which could be explained as a consequence of the decreased venous return during the strain, was already reported in the literature (Inan et al 2008). However, this decrease in venous return was not visible in terms of amplitudes, in contrast to the reduced values of $A_{\mathrm{IJ}}$ reported in Inan et al (2009).

On the other hand, the observed reduction in the RJ interval from standing to supine is likely due to the increase in venous return associated with the supine condition, which is also manifested by a decrease in the pre-ejection period. This is in agreement with what was observed by McCall et al by studying subjects in a standing position on a weighing scale by comparing results on the ground and during microgravity induced by parabolic flight manoeuvre (McCall et al 2014). The increase in venous return as a characteristic of the supine condition, associated also with a larger stroke volume (Frey et al 1994), might also be reflected in the larger peak-to-peak $A_{\mathrm{JK}}$ amplitude as observed in the supine position compared to standing.

These observations demonstrate the complex interaction between intra-thoracic pressure changes acting on venous return, the effect of the hydrostatic pressure gradient elicited by postural conditions, and the autonomic nervous system modulation on the heart rate and peripheral baroreceptor reflex. 
As these contributions cannot be easily disentangled, the availability of normality reference values for each condition to be used for comparison of new subjects assumes particular importance in the potential identification of possible pathologies involving mechano-electrical cardio-pulmonary coupling.

In conclusion, an acquisition protocol for the BCG signal that included different respiratory manoeuvres to be performed in standing and supine positions was implemented. The acquired signal was analyzed by DTW to compute normality ranges in both temporal and amplitude parameters, and to evaluate potential changes induced by the different settings. Results show dependency from respiratory manoeuvre and posture, supporting the need for the definition of separate normality ranges for each of the studied conditions, as well as the importance of applying specific manoeuvres to highlight any pathological response in the computed BCG parameters.

\section{Acknowledgments}

This research was supported by the Italian Space Agency (contract 2013-064-R.0, PI Enrico Caiani). This work was also supported by project DPI2016-75458-R funded by MINECO (Spain) and FEDER, and by Gobierno de Aragón and the European Social Fund (EU) through BSICoS Group (T96). The CIBER in Bioengineering, Biomaterials \& Nanomedicine (CIBER$\mathrm{BBN}$ ) is an initiative of the Instituto de Salud Carlos III (Spain). P-F Migeotte is supported by the Belgian Federal Science Policy Office (BELSPO) via the ESA PRODEX program.

\section{References}

Aunon J I, McGillem C D and Childers D G 1981 Signal processing in evoked potential research: averaging and modeling Crit. Rev. Bioeng. $5323-67$

Caiani E G, Porta A, Baselli G, Turiel M, Muzzupappa S, Pagani M, Malliani A and Cerutti S 2002 Analysis of cardiac left-ventricular volume based on time warping averaging Med. Biol. Eng. Comput. 40 225-33

Cohen A 1986 Biomedical Signal Processing (Boca Raton, FL: CRC Press)

Collis T, Devereux R B, Roman M J, de Simone G, Yeh J-L, Howard B V, Fabsitz R R and Welty T K 2001 Relations of stroke volume and cardiac output to body composition Circulation 103 820-5

Etemadi M, Inan O T, Giovangrandi L and Kovacs G T A 2011 Rapid assessment of cardiac contractility on a home bathroom scale IEEE Trans. Inf. Technol. Biomed. 15 864-9

Frey M A B, Tomaselli C M and Hoffler W G 1994 Cardiovascular responses to postural changes: differences with age for women and men J. Clin. Pharmacol. 34 394-402

Gubner R S, Rodstein M and Ungerleider H E 1953 Ballistocardiography; an appraisal of technic, physiologic principles, and clinical value Circulation 7 268-86

Guyton A C and Hall J E 2006 Textbook of Medical Physiology 11th edn (Philadelphia: Elsevier Saunders)

Hamilton W F, Woodbury R A and Harper H T 1936 Physiologic relationships between intrathoracic, intraspinal and arterial pressures J. Am. Med. Assoc. 107 853-6

He D D, Winokur E S and Sodini C G 2011 A continuous, wearable, and wireless heart monitor using head ballistocardiogram (BCG) and head electrocardiogram (ECG) Annual Int. Conf. IEEE Engineering in Medicine and Biology Society (Boston, MA, 2011) pp 4729-32

Inan O T, Etemadi M, Wiard R M, Giovangrandi L and Kovacs G T A 2009 Robust ballistocardiogram acquisition for home monitoring Physiol. Meas. 30169

Inan O T, Etemadi M, Wiard R M, Kovacs G T A and Giovangrandi L 2008 Non-invasive measurement of Valsalva-induced hemodynamic changes on a bathroom scale ballistocardiograph 2008 30th Annual Int. Conf. IEEE Engineering in Medicine and Biology Society (Vancouver, BC, 2008) pp $674-77$ 
Inan O T et al 2015 Ballistocardiography and seismocardiography: a review of recent advances IEEE J. Biomed. Health Inf. 19 1414-27

Javaid A Q, Ashouri H, Tridandapani S and Inan O T 2016 Elucidating the hemodynamic origin of ballistocardiographic forces: toward improved monitoring of cardiovascular health at home IEEE J. Transl. Eng. Health Med. 4 1-8

Javorka M, Žila I, Javorka K and Čalkovská A 2001 'Respiratory' oscillations of cardiovascular parameters during voluntary apnea Respir. Physiol. 126 251-4

Lewis R P, Rittogers S E, Froester W F and Boudoulas H 1977 A critical review of the systolic time intervals Circulation 56 146-58

Looga R 2005 The Valsalva manoeuvre-cardiovascular effects and performance technique: a critical review Respir. Physiol. Neurobiol. 147 39-49

Martinez J P, Almeida R, Olmos S, Rocha A P and Laguna P 2004 A wavelet-based ECG delineator: evaluation on standard databases IEEE Trans. Biomed. Eng. 51 570-81

McCall C, Stuart Z, Wiard R M, Inan O T, Giovangrandi L, Cuttino C M and Kovacs G T 2014 Standing ballistocardiography measurements in microgravity 2014 36th Annual Int. Conf. IEEE Engineering in Medicine and Biology Society (Chicago, IL, 2014) pp 5180-3

Pinheiro E, Postolache O and Girão P 2010 Theory and developments in an unobtrusive cardiovascular system representation: ballistocardiography Open Biomed. Eng. J. 4 201-16

Porth C J, Bamrah V S, Tristani F E and Smith J J 1984 The valsalva maneuver: mechanisms and clinical implications Heart Lung 13 507-18

Sakoe H and Chiba S 1978 Dynamic programming algorithm optimization for spoken word recognition IEEE Trans. Acoust. Speech Signal Process. 26 43-9

Shin J H, Hwang S H, Chang M H and Park K S 2011 Heart rate variability analysis using a ballistocardiogram during Valsalva manoeuvre and post exercise Physiol. Meas. 321239

Starr I 1965 Progress towards a physiological cardiology. A second essay on the ballistocardiogram Ann. Intern. Med. 63 1079-105

Starr I 1955 Normal standards for amplitude of ballistocardiograms calibrated by force Circulation 11 914-26

Starr I, Rawson A J, Schroeder H A and Joseph N R 1939 Studies on the estimation of cardiac output in man, and of abnormalities in cardiac function Am. Heart J. 18506

Wiard R M 2012 Validation of non-invasive standing arterial stiffness measurements using ballistocardriography and photoplethysmography PhD Thesis Stanford University (https:// searchworks.stanford.edu/view/9688132)

Zhu X, Chen W, Kitamura K and Nemoto T 2012 Comparison of pulse rate variability indices estimated from pressure signal and photoplethysmogram Proc. 2012 IEEE-EMBS Int. Conf. Biomedical and Health Informatics (Hong Kong, 2012) pp 867-70 